\title{
The Impact of Modeling Nuclear Fragmentation on Delivered Dose and Radiobiology in Ion Therapy
}

\author{
Armin Lühr ${ }^{1,2,3}$, David C. Hansen ${ }^{1,2}$, Ricky Teiwes ${ }^{2}$, Nikolai \\ Sobolevsky $^{4}$, Oliver Jäkel ${ }^{5,6}$, and Niels Bassler ${ }^{1,2}$ \\ ${ }^{1}$ Department of Experimental Clinical Oncology, Aarhus University Hospital, \\ Aarhus, Denmark \\ ${ }^{2}$ Department of Physics and Astronomy, University of Aarhus, Aarhus, Denmark \\ ${ }^{3}$ OncoRay - Center for Radiation Research in Oncology, Technische Universität \\ Dresden, Dresden, Germany \\ ${ }^{4}$ Department of Neutron Research, Institute for Nuclear Research of the Russian \\ Academy of Sciences, Moscow, Russia \\ ${ }^{5}$ Department of Medical Physics in Radiation Oncology, German Cancer Research \\ Center (DKFZ), Heidelberg, Germany \\ ${ }^{6}$ Heidelberg Ion Beam Therapy Center (HIT), Heidelberg University Hospital, \\ Heidelberg, Germany \\ E-mail: luehr@phys.au.dk
}

\begin{abstract}
.
The importance of nuclear interactions for ion therapy arises from the influence of the particle spectrum on first, radiobiology and therefore also on treatment planning second, the accuracy of measuring dose and third, the delivered dose distribution. This study tries to determine the qualitative as well as quantitative influence of the modeling of inelastic nuclear interactions on ion therapy. Thereby, three key disciplines are investigated, namely, dose delivery, dose assessment, and radiobiology. In order to perform a quantitative analysis a relative comparison between six different descriptions of nuclear interactions is carried out for carbon ions. The particle transport is simulated with the Monte Carlo code SHIELD-HIT10A while dose planning and radiobiology are covered by the analytic treatment planning program for particles TRiP which determines the relative biological effectiveness (RBE) with the local effect model. The obtained results show that the physical dose distribution can in principle be significantly influenced by the modeling of fragmentation (about 10\% for a $20 \%$ change in all inelastic nuclear cross sections for a target volume ranging from $15 \mathrm{~cm}$ to $25 \mathrm{~cm}$ ). While the impact of nuclear fragmentation on stopping power ratios can be neglected the fluence correction factor may be influenced by the applied nuclear models. In contrast to the results for the physical dose, the variation of the RBE is only small (about $1 \%$ for a $20 \%$ change in all inelastic nuclear cross sections) suggesting a relatively weak dependence of radiobiology on the detailed composition of the particle energy spectrum of the mixed radiation field. Also, no significant change (about 0.2 $\mathrm{mm}$ ) of the lateral penumbra of the RBE-weighted dose is observed.
\end{abstract}




\section{Introduction}

Radiotherapy with ion beams allows in principle for a higher degree of dose conformation to the target volume compared to photons, electrons or neutrons due to their so called inverted dose profile with a distinct dose maximum and sharp dose fall-off, i.e., the Bragg peak. When comparing the lightest ions, protons, with heavier ions, e.g., carbon ions at least three characteristic differences can be named being of relevance to radiotherapy. Heavier ions have first, a significantly reduced lateral scattering leading to steeper lateral dose distributions, second, an increased linear energy transfer (LET) leading to an increased relative biological effectiveness (RBE) towards the Bragg peak region, and third, a higher probability for inelastic nuclear interactions. These nuclear interactions can lead to fragmentation of the projectile or target nuclei. Therefore, they change the particle fluence from a pure beam of primary ions into a mixed beam containing a number of lighter ions and neutrons which may have a broad energy distribution.

The importance of nuclear interactions for ion therapy arises, on the one hand, from the influence of the particle spectrum on radiobiology and therefore also on treatment planning (Krämer et al. 2000, Krämer \& Scholz 2000). A further reason why the impact of modeling nuclear reactions on ion therapy should be considered is the possible influence on the accuracy of measured and delivered dose, i.e., on dosimetry and dose distribution, respectively. In radiotherapy, the effects of ionizing radiation are related to radiation dose. Since both tumor control and normal tissue complication probability strongly depend on dose, prediction of clinical results requires accurate knowledge of the dose deposited in the patient. According to recommendations of the International Commission on Radiological Measurement and Units (ICRU), the dose to the planning target volume (PTV) in the patient should be delivered with an uncertainty of less than 5 $\%$ at the $2 \sigma$ level (ICRU Report 24 1976). As a clinical treatment includes uncertainties due to dose calculation, dose delivery, as well as dose measurement these individual actions should be performed with an uncertainty well below this overall requirement, i.e. in the order of $1 \%$ (Karger et al. 2010).

Following these lines of argumentation, the question might be raised whether the modeling of nuclear interaction is sufficiently accurate in order to fulfill the stated requirements for clinical practice. This question was also recently raised by (Pshenichnov et al. 2010) indicating its relevance, yet they where more concerned about the performance of different models from a physical point of view and did not evaluate the impact in a clinical setting. Accordingly, the purpose of this work may be phrased in the following two questions:

- What is the qualitative influence of nuclear interaction models on radiotherapy?

- How large is the quantitative influence of uncertainties in models on radiotherapy?

In order to answer the first question one has to figure out those key disciplines within ion therapy which are affected by nuclear interactions. The following six subjects are obviously influenced by fragmentation: 
(i) dose delivery (treatment planning)

(ii) dose assessment (dosimetry)

(iii) radiobiology (deterministic effects)

(iv) secondary cancer from neutrons (stochastic effects)

(v) radioprotection (shielding)

(vi) production of radionuclides (PET imaging)

After having identified these disciplines the second question intends to quantify how large an effect can be when changing the modeling of inelastic nuclear interactions. Or to put it in other words, is the variation of, e.g., the dose well below $1 \%$, of the order of $1 \%$ or even much larger due to a different description of the nuclear interaction. The first three topics, (i) - (iii), are discussed in some detail in this work while the latter three, (iv) - (vi), may be subject of a subsequent effort and are partially covered elsewhere, e.g., (Parodi et al. 2007, Hultqvist \& Gudowska 2010).

In the following section the methods used in this study are presented. Section 3 provides results illustrating the influence of changing the modeling of nuclear interaction on dose delivery, radiobiology, and dose assessment. The obtained data are compared and their relevance in view of ion therapy is discussed in section 4 . This paper closes with the conclusions based on this study.

\section{Materials and Methods}

\subsection{SHIELD-HIT10A}

In order to simulate the ion transport in water the Monte Carlo (MC) transport code SHIELD-HIT (Dementyev \& Sobolevsky 1999, Gudowska et al. 2004) was employed in its latest version SHIELD-HIT10A (Hansen et al. 2012b, Hansen et al. 2012a). SHIELDHIT10A is a MC code specialized to ion therapy. This is reflected in the energy range of the physical models and the experiments to which they were calibrated. Additionally, it can exchange output and input files with other programs relevant to ion therapy. Among these are the treatment planning program TRiP (Krämer et al. 2000, Krämer \& Scholz 2000) and the amorphous track model software library libamtrack (Greilich et al. 2010) for which, e.g., energy-resolved fluence spectra and depth-dose distributions can be exported in their native .spc and .ddd format, respectively. Furthermore, a wide range of stopping power data from different tables and programs, which are covered by the software library libdEdx $\ddagger$ described in (Lühr et al. 2012), can be imported into SHIELD-HIT10A. Earlier studies have shown SHIELD-HIT10A's ability regarding ion therapy in the context of dose assessment, namely the determination of stopping power ratios (Lühr et al. 2011a) and fluence correction factors (Lühr et al. 2011b). 


\subsection{Modeling of nuclear fragmentation}

The nuclear fragmentation in SHIELD-HIT10A is handled by the Multi Stage Dynamical Model (MSDM) (Botvina et al. 1997). The MSDM follows a 3-step approach including a fast cascade step, a pre-equilibrium step, and finally a de-excitation model. Different models are available for the three steps and their selection depends on the kind and energy of the interacting particles. In simulations with SHIELD-HIT10A relevant for ion beam therapy the three steps are usually modeled by the Dubna cascade (Toneev \& Gudima 1983), an exciton model (Gudima et al. 1983), and a Fermi-Breakup model (Fermi 1950, Botvina et al. 1987). Very recently, the modeling of nuclear fragmentation used by SHIELD-HIT10A was improved and benchmarked against experimental data as well as compared to other MC codes (Hansen et al. 2012b). This included an update of inelastic nuclear cross sections following recent experimental data as well as a fine tuning of model parameters in the Fermi-Breakup model.

In order to determine the influence of modeling nuclear fragmentation and in particular the recently implemented improvements in SHIELD-HIT10A on ion therapy a relative comparison has been performed in this work. This way, not only qualitative but also quantitative conclusions can be drawn. In total six cases A) - F) have been considered which are listed in table 1 and are briefly described in the following.

In case A), the description of nuclear interactions is used as currently implemented in SHIELD-HIT10A being benchmarked against experimental data. This case serves in this study as a reference relative to which the other results are compared to. In case B), all nuclear interactions are turned off entirely in the MC simulation. Accordingly, no fragments such as lighter ions or neutrons are produced serving thereby as a hypothetical limiting case. In the cases C) and D), the inelastic nuclear cross sections for all combinations of ions and target nuclei are uniformly decreased and increased by 20 $\%$, respectively. These may therefore be understood as a kind of quantitative test for the impact of a possible $20 \%$ error of the inelastic nuclear cross sections on ion therapy.

In the two last cases E) and F), the two model parameter of SHIELD-HIT's Fermi-

$\ddagger d E d x$, a web-based fronted of $l i b d E d x$, is available at http://dedx.au.dk.

Table 1. Specifications of the six different cases of how the modeling of the nuclear interaction is modified relative to the default settings in the SHIELD-HIT10A. Further description of the six cases is given in section 2.2.

\begin{tabular}{cl}
\hline Case & Description \\
\hline A & Reference: nuclear models as in SHIELD-HIT10A. \\
B & Turning off nuclear reactions entirely. \\
C & Decreasing all inelastic cross sections by $20 \%$. \\
D & Increasing all inelastic cross sections by $20 \%$. \\
E & Fermi-Breakup model parameters: $V_{\mathrm{fr}} / V_{0}=1.0$ and $V_{\mathrm{fr}}^{\mathrm{C}} / V_{0}=1.0$. \\
F & Fermi-Breakup model parameters: $V_{\mathrm{fr}} / V_{0}=30.0$ and $V_{\mathrm{fr}}^{\mathrm{C}} / V_{0}=30.0$. \\
\hline
\end{tabular}


Breakup model, the free volume $V_{\text {fr }} / V_{0}$ and the Coulomb volume $V_{\mathrm{fr}}^{\mathrm{C}} / V_{0}$, are changed. These parameters were subject to some discussion, e.g., recently in the context of ion therapy (Pshenichnov et al. 2010, Hansen 2011, Hansen et al. 2012b). Their values are physically motivated but they are only restricted within a relatively large parameter space. Therefore, appropriate values for the Fermi-Breakup parameters have to be calibrated by comparison to experimental data by what these parameters may become a source of uncertainties for fragmentation. In SHIELD-HIT10A they were optimized to reproduce experimental fragmentation data resulting in the values $V_{\text {fr }} / V_{0}=0.65$ and $V_{\mathrm{fr}}^{\mathrm{C}} / V_{0}=18.0$ (Hansen et al. 2012b) which are used in the cases A) - D). The two sets of model parameters used in the two remaining cases are first in case $\mathrm{E}), V_{\mathrm{fr}} / V_{0}=1.0$ and $V_{\mathrm{fr}}^{\mathrm{C}} / V_{0}=1.0$ and second in case $\left.\mathrm{F}\right), V_{\mathrm{fr}} / V_{0}=30.0$ and $V_{\mathrm{fr}}^{\mathrm{C}} / V_{0}=30.0$. The setting of model parameter in case E), which was the default in earlier versions of SHIELD-HIT, leads relative to the improved default values of SHIELD-HIT10A to fewer and larger fragments while case F) leads, on the other hand, to more and smaller fragments.

\subsection{Dose planning and radiobiology}

In this study the treatment planning program TRiP was employed to calculate the RBE and the RBE-weighted dose as well as to perform physical and biological dose optimization for carbon ion beams. TRiP allows for relatively large flexibility regarding the physical description underlying the ion transport. This is achieved by the fact that the physical information needed for optimization, such as depth-dose distributions, energy-resolved particle spectra, and stopping-power data, are read in as external data files. On the other hand, SHIELD-HIT10A is able to directly export physical data sets in the format required by TRiP. Files containing stopping power data in TRiP format can be produced with the help of the stopping power library libdEdx. The multiple scattering option in TRiP was used for dose optimization and subsequent dose calculation in order to obtain meaningful results also in the lateral direction.

This possibility of data exchange between the MC code SHIELD-HIT10A and the treatment planning software TRiP has been used in the current work in the following way: First, depth-dose distributions and energy-resolved particle-spectra files were calculated with SHIELD-HIT10A for mono energetic carbon ion beams in water for 52 different initial energies ranging from $20 \mathrm{MeV} / \mathrm{A}$ to $500 \mathrm{MeV} / \mathrm{A}$. This was done for all six cases A) - F), which are described in section 2.2. The resulting depth-dose distributions and energy-resolved particle spectra were exported as six complete data

Table 2. Specifications of the two spread-out Bragg peaks (SOBP) used in this study which were optimized with the treatment planning program TRiP assuming active beam scanning. All values are given in $\mathrm{mm}$.

\begin{tabular}{lrrrrr}
\hline Name & width & proximal & distal & midpoint & lateral \\
\hline SOBP I & 100 & 150 & 250 & 200 & 30 \\
SOBP II & 50 & 50 & 100 & 75 & 30 \\
\hline
\end{tabular}


sets in TRiP format. Then, a 3D dose optimization was performed with TRiP in order to produce a spread-out Bragg peak (SOBP) for carbon ions in water using the modeling of the nuclear interaction of the reference case A), i.e., the default setting in SHIELDHIT10A. The initial ion distribution (spatial and energetic distribution of the ions in front of the water target) which resulted from the dose optimization was stored in a raster file. It is this file which specifies the ion fluence distribution which has to be delivered by the accelerator unity in order to produce the optimized dose distribution in the target volume. In a next step this initial ion fluence distribution, stored in the raster file, was used as input to recalculate the resulting dose distribution with TRiP for all six cases A) - F) which reflect the different descriptions of the nuclear interactions. The described procedure was performed two times for one SOBP first, on a homogeneous physical dose of 2 Gy and second, for a homogeneous RBE-weighted dose of 2 Gy (RBE). In total two different SOBP were considered which are specified in table 2

The calculation of the RBE-weighted dose requires the knowledge of the RBE. In order to model the radiobiology of ions TRiP uses the local effect model (LEM) (Scholz et al. 1997) here in version I. The LEM requires the full energy-resolved particle spectrum produced by the ion beam and is therefore dependent on the extent of nuclear fragmentation which occurs due to nuclear interactions of the ions.

\subsection{Correction factors for dosimetry}

Dosimetry protocols for clinical purposes are generally formulated in terms of absorbed dose to water in water. Under realistic conditions, however, it is not always possible to assess the dose to water in water. Instead, the dose is often measured in a medium different from water, such as air in the case of air-filled ionization chambers. This difference can be corrected for using the stopping-power ratio (STPR) between water

and the medium $\mathrm{m}$ at the water-equivalent depth $z_{\mathrm{w}-\text { eq }}$. The water-equivalent depth for a particular depth in a medium $\mathrm{m}$,

$$
z_{\mathrm{w}-\mathrm{eq}}=z_{\mathrm{m}} \frac{R_{\mathrm{w}}}{R_{\mathrm{m}}},
$$

can be determined using the ratio of the ion ranges in water and medium $\mathrm{m}, R_{\mathrm{w}}$ and $R_{\mathrm{m}}$, respectively. In this work, the ranges of monoenergetic ions were estimated as the depths distal to the Bragg peak where the dose drops to $50 \%$ of the dose maximum.

The STPR $S_{\mathrm{w} / \mathrm{m}}$ between water and a material $\mathrm{m}$ is given as a particle fluence weighted average over all primary and secondary particles (cf. (IAEA TRS-398 2000)). It is determined by calculating the dose ratio via track-length fluence in water $\Phi_{\mathrm{w}, i}\left(E, z_{\mathrm{w}-\mathrm{eq}}\right)$ of particle type $i$ at $z_{\mathrm{w}-\mathrm{eq}}$ as function of particle energy $E$ and mass stopping power $S_{i}(E) / \rho$ in water as well as in the other material m:

$$
S_{\mathrm{w} / \mathrm{m}}\left(z_{\mathrm{w}-\mathrm{eq}}\right)=\frac{\sum_{i} \int_{0}^{\infty} \Phi_{\mathrm{w}, i}\left(E, z_{\mathrm{w}-\mathrm{eq}}\right)\left(S_{i}(E) / \rho\right)_{\mathrm{w}} \mathrm{d} E}{\sum_{i} \int_{0}^{\infty} \Phi_{\mathrm{w}, i}\left(E, z_{\mathrm{w}-\mathrm{eq}}\right)\left(S_{i}(E) / \rho\right)_{\mathrm{m}} \mathrm{d} E} .
$$

In a more general case, the phantom material may also be different from water. Examples for this situation are graphite calorimetry used as a primary dose standard 
(Palmans et al. 2004) or solid phantoms, such as PMMA, for an increased spatial resolution. Likewise, a water phantom is just a naive model of the human body. Under these conditions one measures dose to medium in medium, $D_{\mathrm{m}}\left(z_{\mathrm{m}}\right)$, at a depth $z_{\mathrm{m}}$. In order to comply with the requirements for dose verification a factor is needed to translate the measured dose to medium in medium to dose to water in water $D_{\mathrm{w}}\left(z_{\mathrm{w}-\mathrm{eq}}\right)$. This factor is defined by the ratio of the two dose values,

$$
\frac{D_{\mathrm{w}}\left(z_{\mathrm{w}-\mathrm{eq}}\right)}{D_{\mathrm{m}}\left(z_{\mathrm{m}}\right)}=\frac{\sum_{i} \int_{0}^{\infty} \Phi_{\mathrm{w}, i}\left(E, z_{\mathrm{w}-\mathrm{eq}}\right)\left(S_{i}(E) / \rho\right)_{\mathrm{w}} \mathrm{d} E}{\sum_{i} \int_{0}^{\infty} \Phi_{\mathrm{m}, i}\left(E, z_{\mathrm{m}}\right)\left(S_{i}(E) / \rho\right)_{\mathrm{m}} \mathrm{d} E},
$$

and it can be rewritten as a product of the STPR and the fluence correction factor $k_{\mathrm{fl}, \mathrm{w} / \mathrm{m}}\left(z_{\mathrm{w}-\mathrm{eq}}\right)$ for water and the medium $\mathrm{m}$

$$
\frac{D_{\mathrm{w}}\left(z_{\mathrm{w}-\mathrm{eq}}\right)}{D_{\mathrm{m}}\left(z_{\mathrm{m}}\right)}=S_{\mathrm{w} / \mathrm{m}}\left(z_{\mathrm{w}-\mathrm{eq}}\right) k_{\mathrm{f}, \mathrm{w} / \mathrm{m}}\left(z_{\mathrm{w}-\mathrm{eq}}\right) .
$$

From that the definition of the fluence correction factor $(\mathrm{FCF})$,

$$
k_{\mathrm{fl}, \mathrm{w} / \mathrm{m}}\left(z_{\mathrm{w}-\mathrm{eq}}\right)=\frac{\sum_{i} \int_{0}^{\infty} \Phi_{\mathrm{w}, i}\left(E, z_{\mathrm{w}-\mathrm{eq}}\right)\left(S_{i}(E) / \rho\right)_{\mathrm{m}} \mathrm{d} E}{\sum_{i} \int_{0}^{\infty} \Phi_{\mathrm{m}, i}\left(E, z_{\mathrm{m}}\right)\left(S_{i}(E) / \rho\right)_{\mathrm{m}} \mathrm{d} E},
$$

follows with the STPR given as in (2). The definition in (5) is the same as in (Palmans et al. 2002) where $\Phi_{\mathrm{m}, i}\left(E, z_{\mathrm{m}}\right)$ is the track-length fluence in medium $\mathrm{m}$ at a depth $z_{\mathrm{m}}$ in the medium.

From the definitions of the STPR and the FCF in (2) and (5), respectively, it follows that both quantities depend on the energy-resolved particle fluence and therefore on nuclear interaction and in principle also on the initial ion energy. While for the STPR only one calculation with the MC code SHIELD-HIT10A in water is sufficient, two calculations, one in water and one in the medium $\mathrm{m}$, are required for the FCF. Here, STPR and FCF are considered for monoenergetic ion beams only since dose measurements in the course of regular quality assurance for active beam delivery is usually performed using monoenergetic beams. A discussion of these correction factors for the case of SOBP can be found elsewhere (Lühr et al. 2011b).

\section{Results}

In this work a relative comparison is performed in order to determine the quantitative dependence of the physical dose distribution, of radiobiology, and of dose assessment on inelastic nuclear interactions and the way they are modeled. Thereby, the following strategy is used. The default settings in SHIELD-HIT10A are considered as reference case A). The results obtained for differently modeled nuclear interactions in the cases B) - F), specified in table 1 , are then considered relative to this reference. An overview of results in quantitative form for all cases A) - F) is also presented in tables 3 and 4 at the end of this section. 

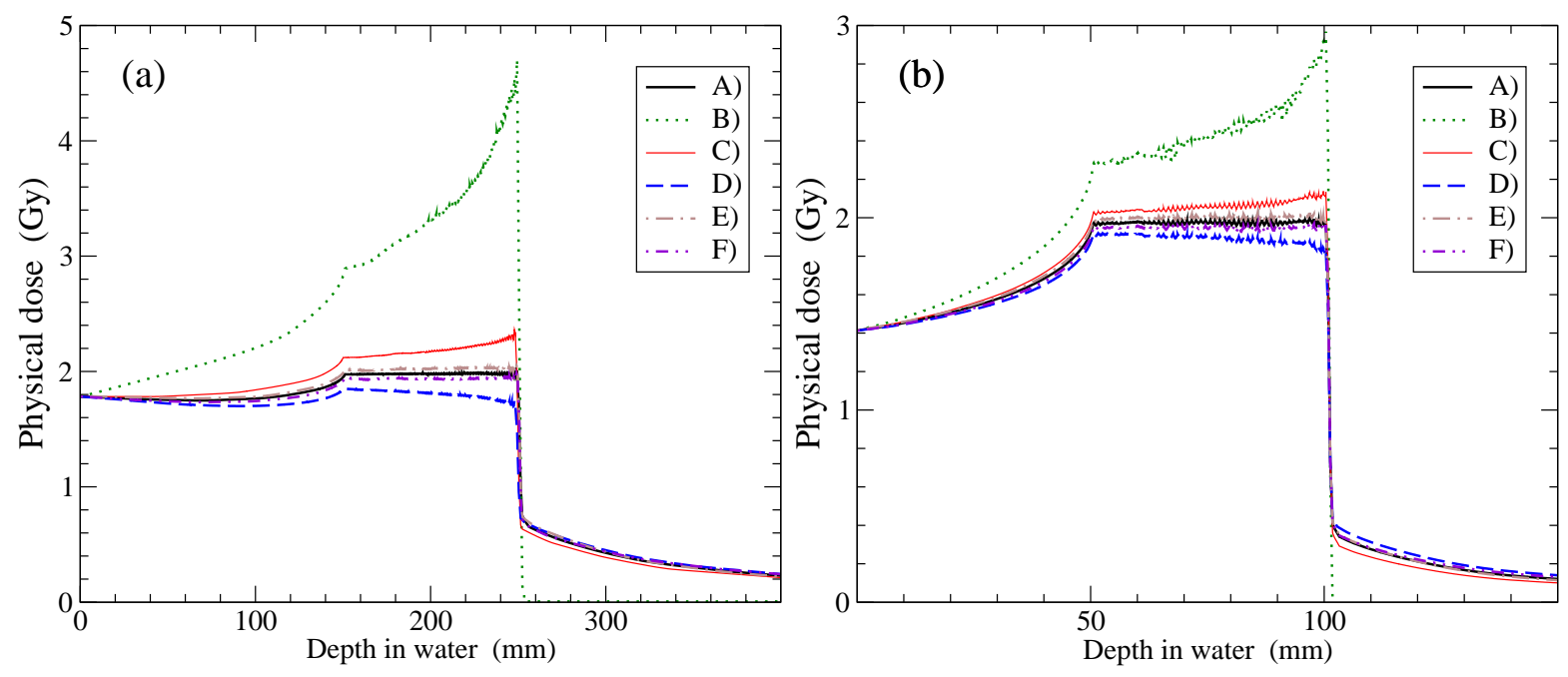

Figure 1. Dose distributions for carbon ions in water using the six different cases A) - F) of modeling nuclear interactions as specified in table 1. The energy fluence distribution of the initial ions is the same for all cases A) - F). It was obtained with TRiP by optimizing for the reference case A) on a SOBP with a homogeneous physical dose level of 2 Gy. (a) SOBP I and (b) SOBP II as specified in table 2.

\subsection{Dose distribution}

The physical dose distributions of carbon ions in water for SOBP I and SOBP II, specified in table 2 , are shown in figure $1 \mathrm{a}$ and $1 \mathrm{~b}$, respectively. The SOBP were optimized with TRiP on a homogeneous 2 Gy level using the reference case A). The physical dose distributions resulting from the same initial ion distribution but using five different ways of modeling the nuclear interaction, cases B) - F), are also shown in figure 1 and are compared to the reference A) in the following.

First, SOBP I is considered. Turning off the nuclear reactions entirely, i.e. case B), results in a drastic increase of the physical dose relative to case A) in the SOBP region from $45 \%$ up to $125 \%$ at the proximal and distal edge of SOBP I, respectively. But on the other hand, the dose is zero beyond the SOBP since in case B) no fragments are produced, due to the missing nuclear interactions, which could form a fragmentation tail. In both cases C) and D), i.e. a $20 \%$ decrease and increase of the inelastic nuclear cross sections, the physical dose is increased and decreased, respectively, by nearly the same factors. At the beginning of SOBP I the difference to the reference A) is about $6.5 \%$ and it becomes as large as $15 \%$ towards the end of SOBP I. For depths beyond SOBP I the curves for C) and D) are about $5 \%$ lower and higher, respectively, than the reference A). The changes of the model parameters in the Fermi-breakup model in the cases E) and F) lead to fewer but larger fragments and a $2.5 \%$ increase of dose and to more but smaller fragments and a $2.5 \%$ decrease of dose, respectively, in the SOBP region. In the region distal to SOBP I the deviations of the dose for E) and F) from A) vary between 0 and $5 \%$ being both partly smaller and larger than the reference $\mathrm{A}$ ).

The results for SOBP II in figure $1 \mathrm{~b}$ agree qualitatively with those presented for 

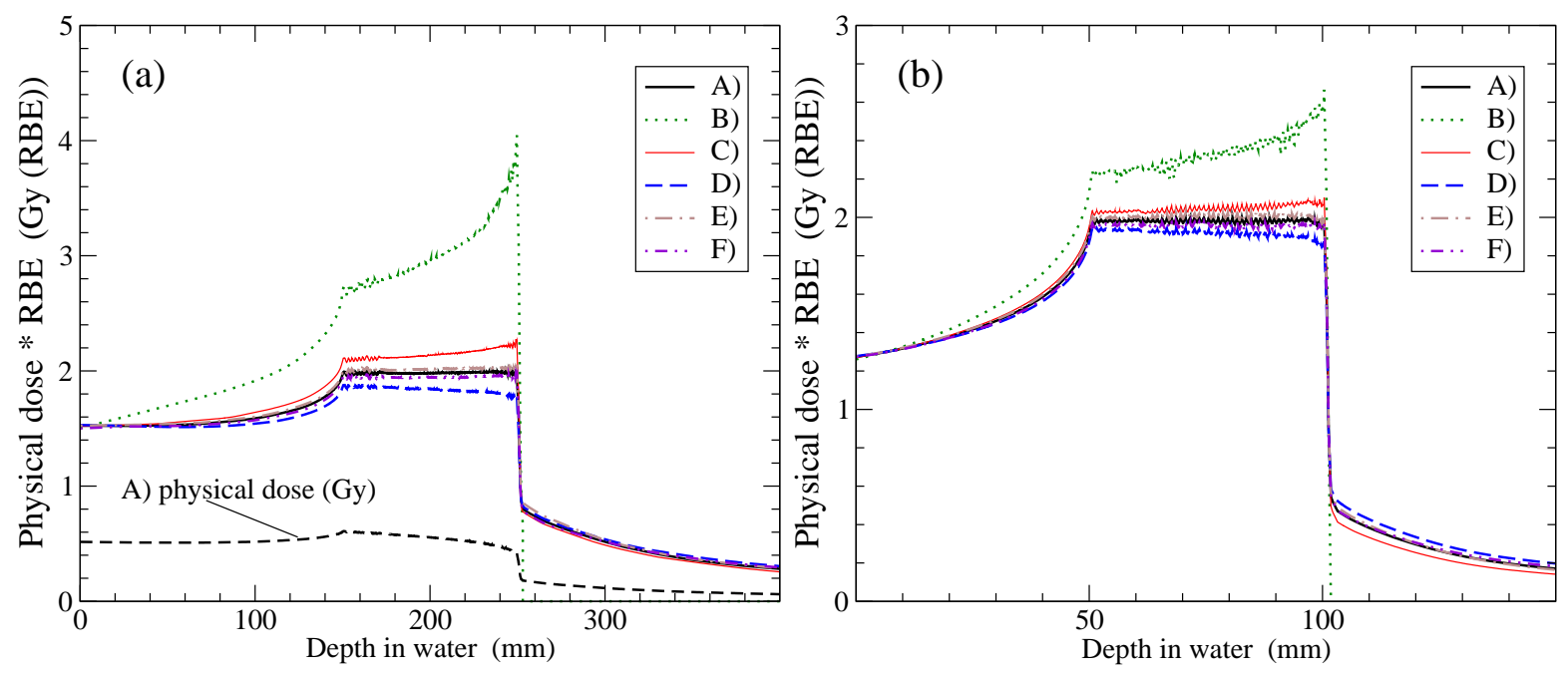

Figure 2. As figure 1 but optimized on a homogeneous RBE-weighted dose level of 2 Gy (RBE). (a) SOBP I and (b) SOBP II as specified in table 2.

SOBP I. However, the quantitative deviations between A) and the cases B) - F) are in general smaller by a factor of 2.5 - 3 for the smaller and less deep SOBP II.

\subsection{Radiobiology}

The RBE-weighted dose distributions of carbon ions in water for SOBP I and SOBP II are shown in figure $2 \mathrm{a}$ and $2 \mathrm{~b}$, respectively. Both SOBP were biologically optimized with TRiP on a homogeneous 2 Gy (RBE) level based on case A). The reference case A) is compared to the RBE-weighted dose distributions resulting from the same initial ion distribution but using the five cases B) - F) for modeling the nuclear interaction as specified in table 1. Additionally, the physical dose distribution of SOBP I for the reference case A) is shown in figure 2 a.

First, SOBP I is considered. Similar to what was observed for the physical dose for SOBP I in figure 1a, case B) results in a pronounced increase of the RBE-weighted dose of about $35 \%$ at the beginning, about $100 \%$ at the distal end of the SOBP region and no dose beyond. In the cases C) and D), the RBE-weighted dose is increased and decreased, respectively, in practically the same way as it has been observed before for the physical dose in figure 1 . At the beginning of SOBP I the difference to the reference A) is about $5 \%$ and it becomes as large as $13.5 \%$ towards the end of SOBP I. For depths beyond SOBP I, the curves for C) and D) are about $5 \%$ lower and higher, respectively, than the reference $\mathrm{A}$ ). In the cases $\mathrm{E}$ ) and $\mathrm{F}$ ) a $2 \%$ increase and a $2 \%$ decrease, respectively, of the RBE-weighted dose can be observed in the SOBP region. Beyond SOBP I, the differences of these two curves from reference A) are about $5 \%$.

The results for SOBP II in figure $2 \mathrm{~b}$ agree again qualitatively with those for SOBP I. The quantitative deviations between A) and the cases B) - F) are also once again smaller by a factor of about 2.5 for SOBP II compared to SOBP I (cf. table 3) .

In figure 3 , the $\mathrm{RBE}$ distributions for carbon ions in water are presented for the 

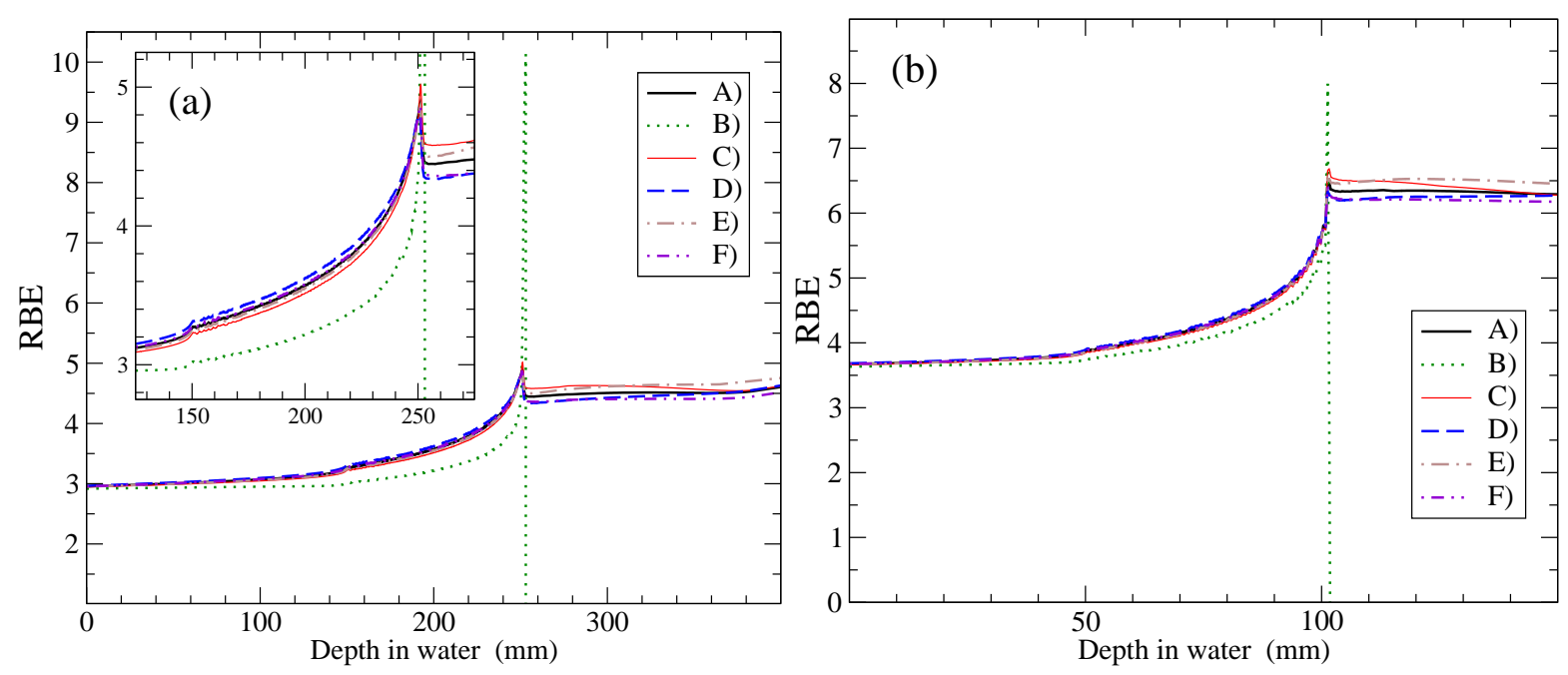

Figure 3. RBE distributions for carbon ions in water for the RBE-weighted dose distributions of the six cases A) - F) shown in figure 2. The inset shows the SOBP region on an enlarged scale. (a) SOBP I and (b) SOBP II.

RBE-weighted dose distributions of the six cases A) - F) shown in figure 2. First, results for SOBP I are considered shown in figure 3a. The RBE in case A) increases from about 3.25 at the proximal edge of SOBP I to nearly 5 at the distal edge. All RBE curves are very similar to the reference case A) except for the case B). For the latter case, the RBE values are about $10 \%$ and $15 \%$ smaller than for case A) at the beginning of SOBP I and towards its distal end, respectively. However, a distinct RBE peak with a value of 10.1 is observed around $252 \mathrm{~mm}$, i.e. shortly after the distal edge of SOBP I and a RBE of zero for larger depths in case B). For the four other cases C) - F), the difference to $\mathrm{A}$ ) is smaller than $1.5 \%$ in the SOBP region where the $\mathrm{RBE}$ values for $\mathrm{C}$ ) and $\mathrm{D}$ ) are smallest and largest, respectively. Beyond the SOBP region, the differences to A) are a bit larger and of the order of $3 \%$.

For SOBP II, shown in figure $3 \mathrm{~b}$, the RBE in case A) increases from about 3.9 at the proximal edge of SOBP II to 5.8 at the distal edge. As seen before for the physical and RBE-weighted dose, also the RBE results for SOBP II agree qualitatively with those for SOBP I. Again, for SOBP II the deviations among the six cases A) - F) are in general by a factor of about 2.5 smaller than those of SOBP I.

\subsection{Lateral distribution}

The lateral RBE-weighted dose distribution for SOBP I is shown in figure 4a for the case A) at four different depths. It can be seen that the lateral profile does not change a lot within the first $50 \mathrm{~mm}$ of the SOBP region. In contrast, at a depth of $245 \mathrm{~mm}$, that is $5 \mathrm{~mm}$ prior to the distal edge, a broadening of the lateral distribution is clearly observable. However, the RBE-weighted dose in the target at a depths of $245 \mathrm{~mm}$ is comparable to that at the proximal part of the SOBP between $150 \mathrm{~mm}$ and $200 \mathrm{~mm}$ and only slightly reduced towards the lateral edges of the SOBP region. 

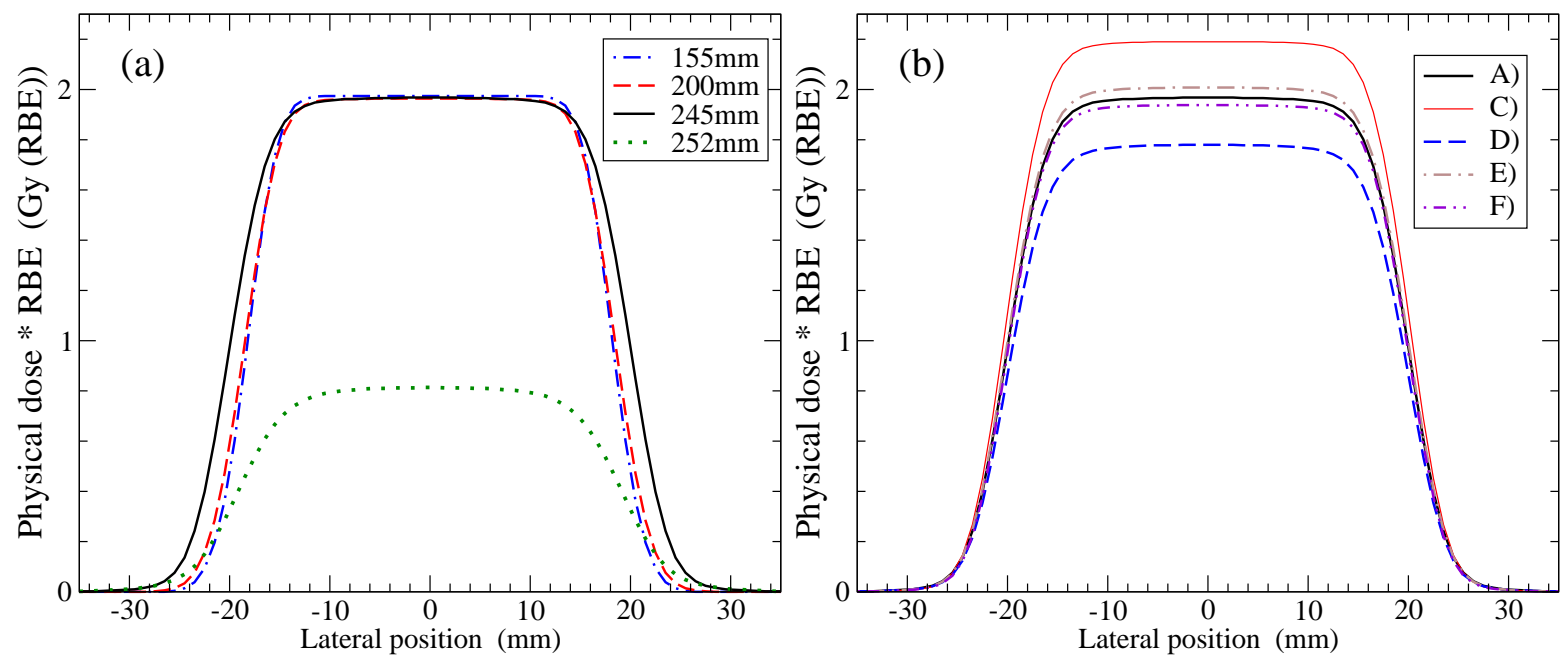

Figure 4. Lateral RBE-weighted dose distributions for SOBP I as presented in figure 2a. (a) Only case A) at four different depths in water. (b) The five cases A) - F) without B) all at a depth of $245 \mathrm{~mm}$ in water.

Figure $4 \mathrm{~b}$ shows the lateral RBE-weighted dose for the five cases A) - F) without B) at a depth in water of $245 \mathrm{~mm}$. It can be seen that the relative differences in dose between the cases, observed before in figure $2 \mathrm{a}$ as a function of depth, range over the whole lateral SOBP region. Only small differences in the lateral penumbra, which is here defined as the distance between the positions of $80 \%$ and $20 \%$ of the dose level within the target, are found for the considered cases and listed in table 3. At a depth of $245 \mathrm{~mm}$ the maximal deviation of the lateral position of $80 \% \mathrm{RBE}$-weighted dose from case A) is well below $0.2 \mathrm{~mm}$ while the penumbra varies in the order of $0.1 \mathrm{~mm}$.

In figure 5 the lateral RBE distributions at a depth of $245 \mathrm{~mm}$ are presented for the RBE-weighted dose curves in figure 4b. All RBE curves vary only little within the lateral extension of the target region. The relative differences between the RBE curves observed before in figure 3 a stretch therefore across the whole SOBP. An increase of $\mathrm{RBE}$ can be observed for a lateral distance $>15 \mathrm{~mm}$ from the center with a maximal $\mathrm{RBE}$ at around $25 \mathrm{~mm}$. The out of field RBE values vary considerably with depth (not shown here) and the RBE maximum seen in figure 5 tends to be much smaller at smaller and larger depths. For a depth of $245 \mathrm{~mm}$ the relative difference between the $\mathrm{RBE}$ curves of the considered cases remain rather small being largest around the RBE maximum with about $3 \%$.

\subsection{Dosimetry}

The stopping power ratio between water and air, as defined in (2), for a $270 \mathrm{MeV} / \mathrm{A}$ carbon beam in water is shown in figure 6 for the six different cases A) - F). The differences among the six curves are in general small. Before the Bragg peak the largest deviations can be found between the reference case A) and case B) with nuclear interactions turned off which can be up to $0.05 \%$. The maximal deviations of the other 


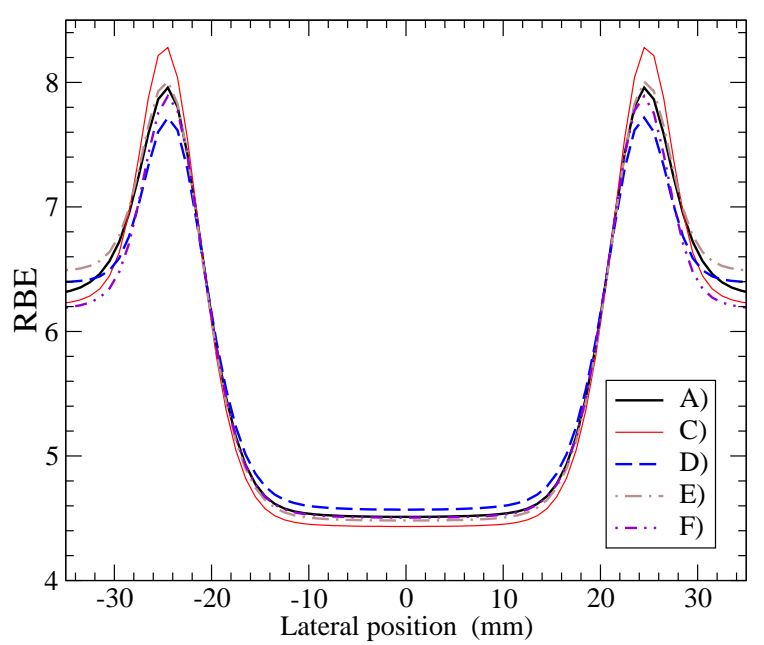

Figure 5. Lateral RBE distributions for carbon ions in water at a depths of $245 \mathrm{~mm}$ for the five RBEweighted dose distributions shown in figure $4 \mathrm{~b}$.

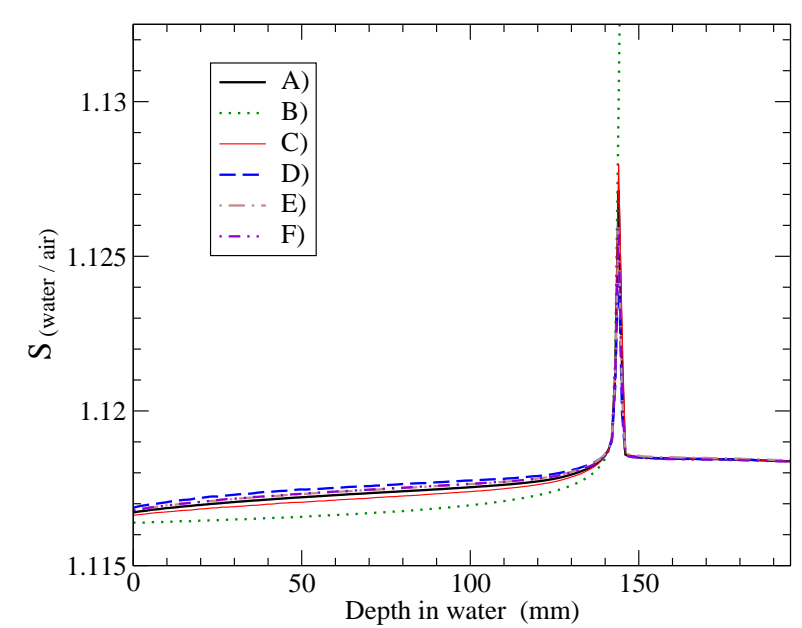

Figure 6. Stopping power ratios between water and air for a 270 $\mathrm{MeV} / \mathrm{A}$ carbon beam in water. The data sets were calculated with SHIELD-HIT10A for the six cases A) - F) of modeling nuclear interaction (cf. table 1).

four curves C) - F) from the reference A) are below $0.02 \%$. Beyond the Bragg peak these four curves differ by less than $0.005 \%$ from the reference while curve B) does not continue since no light fragments are produced without nuclear interaction and all primary particles are stopped at the Bragg peak.

Figure 7 presents FCF, as defined in (5), for a $270 \mathrm{MeV} / \mathrm{A}$ carbon beam for the six different cases A) - F). FCF between water and graphite and between water and PMMA are shown in figure $7 \mathrm{a}$ and $7 \mathrm{~b}$, respectively.

For graphite, the deviation of the FCF for a $270 \mathrm{MeV} / \mathrm{A}$ carbon beam is in general not very pronounced (Lühr et al. 2011b). For the reference case A), it decreases from 0.9985 at zero depth to 0.996 and to 0.96 around $100 \mathrm{~mm}$ and $140 \mathrm{~mm}$, respectively. For case B), that is without nuclear fragmentation, the FCF remains basically unity for all depths before the Bragg peak resulting in a difference to A) of up to $5 \%$ around $140 \mathrm{~mm}$. The largest deviation from the reference A) for the remaining cases can be observed for case F) in which more but smaller fragments are produced. The relative difference increases from 0 at zero depth to $0.55 \%$ and to $1 \%$ around $100 \mathrm{~mm}$ and 140 $\mathrm{mm}$, respectively. All curves of the remaining cases differ at most about $0.15 \%$ and below $1 \%$ at these two depths.

The FCF between water and PMMA are known to deviate only little from unity for all beam energies relevant for ion therapy (Lühr et al. 2011b). For the reference case A), the FCF increases linearly with depth to 1.006 at $100 \mathrm{~mm}$ and from a depth larger than $130 \mathrm{~mm}$ until the Bragg peak it decreases to 0.99. As was observed for graphite, the FCF for case B) remains basically unity for depths proximal to the Bragg peak position. The remaining curves of the cases C) - F) follow practically the reference case and differ 

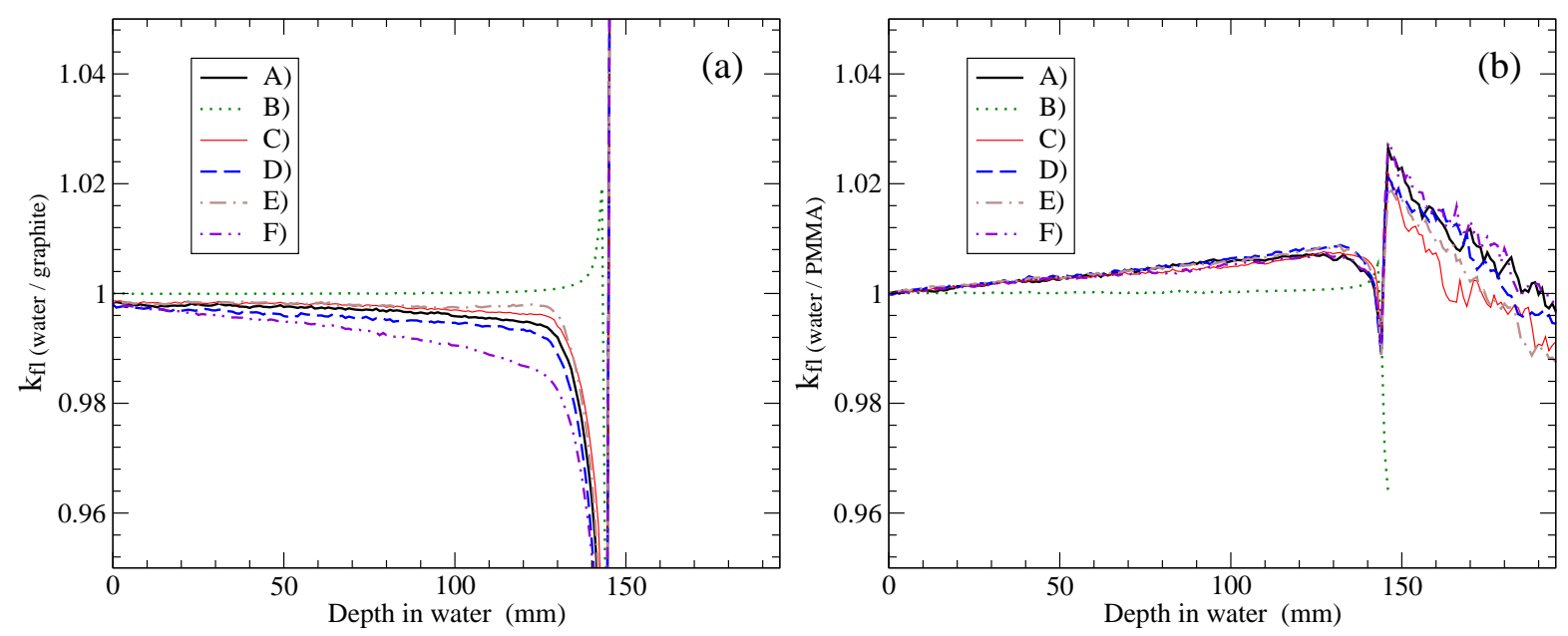

Figure 7. Fluence correction factors for a $270 \mathrm{MeV} / \mathrm{A}$ carbon beam between a) water and graphite and b) water and PMMA. The data sets were calculated with SHIELDHIT10A using the six cases A) - F) of modeling nuclear interaction (cf. table 1).

less then $0.2 \%$ before the Bragg peak with case F) being closest to reference A).

\section{Discussion}

\subsection{Impact on dose distribution}

Based on the results obtained in section 3.1 it may be concluded that the physical dose distribution in a clinical setting can significantly depend on the description of the nuclear interaction. A hypothetical error of, e.g., $20 \%$ in all nuclear cross sections when modeling nuclear interactions may lead for a large and deep seated target volume such as SOBP I to a dose change of about $10 \%$ in the SOBP region. A drastic change of the Fermi-Breakup model parameters, $V_{\mathrm{fr}} / V_{0}$ and $V_{\mathrm{fr}}^{\mathrm{C}} / V_{0}$, which have to be calibrated by comparison to experimental data and which were adapted in the recent version of SHIELD-HIT10A (Hansen et al. 2012b), is, however, less severe. It results for the chosen values in $\mathrm{E}$ ) and $\mathrm{F}$ ) in a change of below $2.5 \%$ in the SOBP region of SOBP I.

For a smaller and shallower target volume, such as SOBP II, the deviations are systematically smaller by about a factor of 2.5 compared to SOBP I. This can be explained by the fact that the ions experiences less nuclear interactions since they have lower energies and traverse a shorter distance in the target material. That means that inaccuracies in the modeling of nuclear reactions are of less importance for small target volumes close to the surface while they can have a significant impact $(>1 \%)$ on the dose distribution for large and deep-seated target volumes.

At the same time it should be said that the physical dose can be usually accessed experimentally with an uncertainty of less the $4 \%$ (IAEA TRS-398 2000). Accordingly, a large error in the total nuclear cross sections of a MC code and consequently also in absorbed dose, as reported above for SOBP I, can be prevented by relatively simple experimental dose verification. Accordingly, the uncertainties of inelastic nuclear cross 
Table 3. Deviations in percent relative to the reference case A) as presented in sections 3.1 to 3.3 for absorbed dose, RBE-weighted dose, RBE, and lateral penumbra of the RBE-weighted dose for the cases B) - F) (cf. table 1). See text for explanations. For case A) the corresponding absolute values are given. Data are provided for the SOBP regions of SOBP I and II and lateral at a depth of $245 \mathrm{~mm}$ for SOBP I.

\begin{tabular}{|c|c|c|c|c|c|}
\hline \multirow[t]{2}{*}{ Case } & \multirow[t]{2}{*}{ Dose } & \multirow[t]{2}{*}{ Dose $\times \mathrm{RBE}$} & \multirow[t]{2}{*}{$\mathrm{RBE}$} & \multicolumn{2}{|c|}{ Lateral Dose $\times$ RBE } \\
\hline & & & & Penumbra & Position $80 \%$ \\
\hline \multicolumn{6}{|c|}{ SOBP I } \\
\hline A & 2 Gy & 2 Gy (RBE) & $3.25-5$ & $5.25 \mathrm{~mm}$ & $17.28 \mathrm{~mm}$ \\
\hline B (\%) & $45-125$ & $35-100$ & $-10--15$ & & \\
\hline $\mathrm{C}(\%)$ & $6.5-15$ & $5-13.5$ & -1.5 & -1.9 & 0.8 \\
\hline $\mathrm{D}(\%)$ & $-6.5--15$ & $-5--13.5$ & 1.5 & 2.1 & -0.9 \\
\hline E $(\%)$ & 2.5 & 2 & -0.5 & 1.0 & -0.2 \\
\hline $\mathrm{F}(\%)$ & -2.5 & -2 & 0.5 & -1.9 & 0.2 \\
\hline \multicolumn{6}{|c|}{ SOBP II } \\
\hline A & 2 Gy & 2 Gy (RBE) & $3.87-5.8$ & & \\
\hline B $(\%)$ & $15-48$ & $12-33$ & $-3--6.5$ & & \\
\hline $\mathrm{C}(\%)$ & $2.5-7$ & $2-5$ & -0.5 & & \\
\hline $\mathrm{D}(\%)$ & $-2.5--7$ & $-2--5$ & 0.5 & & \\
\hline E $(\%)$ & 1 & 0.8 & -0.25 & & \\
\hline $\mathrm{F}(\%)$ & -1 & -0.8 & 0.25 & & \\
\hline
\end{tabular}

sections in standard MC codes are usually smaller than $20 \%$.

It is noted that energy conservation holds also for case B) in figure 1 for which no particles are transported beyond the distal edge of the SOBP. That means for SOBP I that all energy is deposited within a depth of $253 \mathrm{~mm}$. In the reference case A), for comparison, the integrated deposited energy from zero depth up to $253 \mathrm{~mm}$ is less than $65 \%$ of the total kinetic energy of the initial ion beam. Hence, more than $35 \%$ of the total energy is deposited beyond the target volume, e.g., in the fragmentation tail. This tail consists mainly of neutrons and light ions, such as protons, with a long ranges. It is much larger than the maximal depth presented in figure 1a. At a depth of $750 \mathrm{~mm}$ in water beyond the distal edge of SOBP I the absorbed energy is still larger than $1 \%$ of that in the SOBP region.

The results also clearly show that fewer and larger fragments, i.e., the cases B), C), and E) lead in general to an increase of dose proximal to the distal edge of the SOBP and a decrease beyond it. The situation is vice versa for more and smaller fragments, i.e., the cases D) and F). This can be explained by the fact that lighter fragments may have longer ranges. In the case of ions this is due to the dependence of the stopping power on the square of the particle charge $Z^{2}$ while neutrons have no charge at all. 
Table 4. Deviations in percent relative to the reference case A) as presented in section 3.4 for STPR, FCF for graphite, and FCF for PMMA for the cases B) - F). For case A) the corresponding absolute values are given. Data for a mono energetic $270 \mathrm{MeV} / \mathrm{A}$ beam at the penetration depth $100 \mathrm{~mm}$ in water are considered.

\begin{tabular}{lccc}
\hline Case & STPR & FCF $_{\text {graph }}$ & FCF $_{\text {PMMA }}$ \\
\hline A & $\mathbf{1 . 1 1 7 5}$ & $\mathbf{0 . 9 9 6}$ & $\mathbf{1 . 0 0 6}$ \\
B (\%) & -0.05 & 0.4 & -0.6 \\
C (\%) & -0.015 & 0.1 & -0.1 \\
D (\%) & 0.02 & -0.1 & 0.06 \\
E (\%) & 0.01 & 0.15 & -0.005 \\
F (\%) & 0.01 & -0.55 & -0.05 \\
\hline
\end{tabular}

\subsection{Impact on radiobiology}

For the clinical application of ion beams, the RBE-weighted dose is of relevance rather than the physical dose distribution and therefore also the underlying radiobiology is of importance. The central finding of this work is that the influence of the description of nuclear interactions on the modeling of radiobiology is rather small when applying the LEM. This statement is nicely verified by comparing the results for the physical dose, RBE-weighted dose, and RBE in figures 1, 2, and 3, respectively. The obtained findings may allow for the hypothesis that the detailed composition of the energyresolved fragment spectrum has in general a limited impact on radiobiology modeled by the LEM. This might in particular be the case in view of the uncertainties in available experimental RBE values which are even for in vitro data often of the order of $20 \%$ (Sørensen et al. 2011) in contrast to the here observed changes in RBE of about $1 \%$.

When comparing the results for the physical dose and the RBE-weighted dose they only clearly differ for the purely theoretical case B), i.e., without nuclear interactions. This is the only case where the physical dose is completely different from the reference A) leading to a significantly changed RBE. For the other four cases C) - F) of modeling the nuclear interaction the deviations to the reference A) for the RBE-weighted dose differ only little from those obtained for the physical dose being of the order of $1 \%$ up to the distal edge of the SOBP. The deviations from the reference A) tend to be a bit smaller for the RBE-weighted dose than for the physical dose.

The RBE depends on the physical quantities dose and LET, determined by the radiation field. Radiation with high LET is usually characterized by an increased RBE while an increase of dose, on the other hand, leads to a decrease of RBE. The observed relative decrease and increase of up to about $1 \%$ for a $20 \%$ change in the nuclear cross sections is most likely caused by the relative increase and decrease, respectively, of the physical dose as seen in figure 1. The small changes in RBE mean, that the changes in dose and LET, originating from the changed particle spectra in the cases C) - F) are not pronounced enough in order to lead to a significantly different RBE. A possible explanation could be that the primary carbon ions dominate the physical quantities 
relevant for the RBE while the detailed changes in the fragmentation spectrum are mostly of minor importance. It might be recalled that the LET scales with the charge of the ion squared $Z^{2}$ leading to a significant impact of the heaviest ions on the doseaveraged LET distribution in comparison to lighter fragments.

Regarding the lateral RBE-weighted dose distribution it can be concluded that the different cases of modeling nuclear fragmentation do not show a significant impact. Figure 4a shows that a good dose coverage is achieved in the whole target volume using the multiple scattering (ms) mode in TRiP. Due to the use of the ms mode a moderate but still significant increase of the lateral penumbra can be observed for depths close to the distal edge of the SOBP while the dose level in the target remains homogeneous. The observed effect for the performed variations of nuclear modeling on the lateral RBEweighted dose is small. Even around the distal edge of a deep-seated target, such as SOBP I, the penumbra varies only by about $0.2 \mathrm{~mm}$ and therefore not significantly for ion therapy. Also, a maximum in the lateral RBE distribution about $10 \mathrm{~mm}$ away from the lateral edge in figure 5 at a depth close to the distal edge of the SOBP has minor impact on RBE-weighted dose since the absorbed dose is small in this region.

The results obtained for the lateral distributions may be understood when considering the particle spectra in more detail. It is known that at large depths in water, as considered in figures 4 and 5, many light fragments exist relative to the number of primary ions (Haettner et al. 2006, Hansen et al. 2012b). However, they do not contribute very much to the lateral penumbra since they have a low $Z$ and therefore a small local energy deposition compared to the primary ions. Furthermore, they deposit their energy in a much larger volume as for example the dose tail beyond SOBP I which comprises more than $30 \%$ of the total initial kinetic energy of the primary carbon ions. On the other hand, some carbon ions which underwent multiple scattering may especially at large depths stop in the lateral vicinity of the target volume depositing dose with a high LET. The high LET of the stopping ions together with the overall low out of field dose may lead to the observed lateral RBE maximum which is highest for depths close to the distal edge of the SOBP. Consequently, the lateral penumbra for the studied carbon fields seem to be more influenced by multiple scattering, i.e., atomic interactions than the distribution of light fragments depending on nuclear interactions.

It should be noted, however, that the current study is focusing on the influence of inelastic nuclear cross sections and the Fermi-Breakup model as well as on a limited number of tissues for the RBE dependence and only on carbon ions. Furthermore, it was assumed that the LEM is able to reproduce the radiobiology of ions.

\subsection{Impact on dosimetry}

The STPR is for all practical reasons purely influenced by atomic physics and the impact of nuclear fragmentation can, on the other hand, be neglected. This statement is verified by first, the results in figure 6 in which the curves for the six different cases A) - F) differ by less than $0.05 \%$ and second, by detailed studies on STPR which were 
recently performed (e.g., (Lühr et al. 2011a, Henkner et al. 2009)). In the work by (Lühr et al. 2011a) it was observed that the STPR mostly depends on the stopping power as a function of residual range of the primary ion as well as the choice of the specific stopping power data used for its calculation. The negligible influence from secondary particles on the STPR makes it possible to establish a radiation quality $Q$ also for ions, namely the residual range in water, as it has been done for protons, e.g., in the IAEA report (IAEA TRS-398 2000).

A recent study of FCF for different ions and target mediums showed that the form of a FCF curve as a function of depth can vary strongly with the ion species and target material (Lühr et al. 2011b). And even if these two parameters are fixed a non-trivial dependence on the initial ion energy can be observed which seem to be determined by the detailed nuclear interactions (Palmans et al. 2002). Therefore, the results obtained in this work may help to formulate a qualitative trend rather than providing a general and quantitative statement. While PMMA has in general small FCF for graphite, this is especially the case for carbon ions with an initial beam energy of $270 \mathrm{MeV} / \mathrm{A}$ compared to beam energies of, e.g., $100 \mathrm{MeV} / \mathrm{A}$ and $400 \mathrm{MeV} / \mathrm{A}$ (Lühr et al. 2011b). These two examples in figure 7 were chosen here because of their potential relevance for dosimetry regarding ion therapy (Karger et al. 2010, Palmans et al. 2004, Palmans et al. 2002).

It is obvious that the FCF depends decisively on nuclear interactions. Turning them off, i.e. case B), results in a factor which is unity, that is no correction, since the fluence remains practically unchanged up to a depth close to the Bragg peak. This behavior is found for all materials and ions as well as energy combinations. When considering the other four cases C) - F) the outcome can depend on the description of nuclear models. The extend, however, depends very much on the combination of target and ion as well as the initial energy of the latter. A good example for this observation is case F). The corresponding FCF curve for graphite deviates most pronounced from case A) while it is, on the other hand, closest to the reference curve for PMMA. In order to be able to quantify these mutual dependencies more thoroughly it would be necessary to perform a detailed study on this subject which, however, is beyond the scope of this work.

Finally, it might be summarized that in order to translate a dose measured in a medium to the corresponding dose in water the product of the STPR and the FCF can be used as multiplicative factor as shown in (3). The meaning of the two correction factors can be expressed in the following way. The STPR corrects for the differences of the electromagnetic interaction between the medium and water while the FCF takes care of the deviations which originate from different nuclear interactions.

\section{Conclusions}

The purpose of this work has been to figure out for which disciplines within ion therapy the modeling of nuclear interactions may have an influence and how pronounced it may be considering clinical conditions. A quantitative study was performed by relative comparison of calculations within the disciplines dose delivery, radiobiology, and dose 
assessment using six different descriptions of the nuclear interactions. The modeling of the physical quantities, such as nuclear interaction, and the ion transport was done with the Monte Carlo code SHIELD-HIT10A while dose planning and the determination of the relative biologic effectiveness (RBE) were performed with TRiP.

A significant impact of how the nuclear interactions are modeled can be identified for the case of dose delivery. An uncertainty of, e.g., the inelastic nuclear cross sections of 20 $\%$ can lead to a change of the physical dose distribution of more than $10 \%$ in the spreadout Bragg peak region for a large and deep-seated target volume. However, these large dose changes may be detected and corrected for by comparison to experimental data. Also, the impact of the uncertainties in nuclear modeling decreases gradually for smaller and shallower target volumes. On the other hand, only a weak influence on radiobiology is observed with a deviation of the RBE mostly below $1 \%$ in the whole target volume. This is in contrast to the large uncertainties connected with determination and modeling of RBE which are assumed to be larger than $10 \%$. One may therefore conclude that under clinically relevant conditions the dependence of the RBE on the details of the particle spectrum of a mixed radiation field is only weak. The lateral penumbra of the RBE-weighted dose seems to be more influenced by multiple scattering of the primary ions than by lighter fragments produced by nuclear interactions.

Regarding dosimetry, the tiny deviations of the stopping power ratio for different modeling of nuclear interactions are too small to be of relevance for the overall accuracy of ion therapy especially in view of the uncertainties attached to stopping power data. For fluence correction factors, in some cases a significant dependence on the description of the nuclear interaction can be observed which is, however, rather diverse and varies non-linearly with the target material and the type and initial energy of the ion.

This study has exclusively focused on the variation of inelastic cross sections and the Fermi-breakup model using only carbon ions as well as LEM I and a limited number of tissues regarding the RBE dependence. Further research within this topic may also contain investigations of other nuclear models as well as the use of realistic patient plans.

\section{Acknowledgments}

The authors very much acknowledge technical advice with the TRiP code and fruitful discussions with Michael Krämer as well as Gheorghe Iancu. This work is supported by the Danish Cancer Society, CIRRO - The Lundbeck Foundation Centre for Interventional Research in Radiation Oncology and by ULICE - Union of Light Ion Centres in Europe.

\section{References}

Botvina A, Dementyev A, Smirnova O, Sobolevsky N \& Toneev V 1997 International Codes and Model Intercomparison for Intermediate Energy Activation Yields, (by R. Michel, P. Nagel), NSC/DOC (97)-1, NEA/PETT 14. 
Botvina A S, Iljinov A S, Mishustin I N, Bondorf J P, Donangelo R \& Sneppen K 1987 Nuclear Physics A 475(4), $663-686$.

Dementyev A V \& Sobolevsky N M 1999 Radiat. Meas. 30(5), 553-557.

Fermi E 1950 Progress of Theoretical Physics 5(4), 570-583.

Greilich S, Grzanka L, Bassler N, Andersen C \& Jäkel O 2010 Radiat. Meas. 45(10), 1406 - 1409. PROCEEDINGS OF THE 11TH SYMPOSIUM ON NEUTRON AND ION DOSIMETRY, iThemba Laboratory for Accelerator-Based Sciences.

Gudima K K, Mashnik S G \& Toneev V D 1983 Nuclear Physics A 401(2), 329 - 361.

Gudowska I, Sobolevsky N, Andreo P, Belkić D \& Brahme A 2004 Phys. Med. Biol. 49, 1933-1958.

Haettner E, Iwase H \& Schardt D 2006 Radiation protection dosimetry 122(1-4), 485.

Hansen D C 2011 Improving nuclear models in SHIELD-HIT for heavy ion therapy Master's thesis Aarhus University.

Hansen D C, Lühr A, Herrmann R, Sobolevsky N \& Bassler N 2012 a Int. J. Rad. Biol. 88(1-2), 195-199.

Hansen D C, Lühr A, Sobolevsky N \& Bassler N $2012 b$ Phys. Med. Biol. 57, 2393.

Henkner K, Bassler N, Sobolevsky N \& Jäkel O 2009 Med. Phys. 36, 1230-5.

Hultqvist M \& Gudowska I 2010 Phys. Med. Biol. 55(22), 6633.

IAEA TRS-398 2000 Absorbed dose determination in external beam radiotherapy an international code of practice for dosimetry based on standards of absorbed dose to water Technical report International Atomic Energy Agency.

ICRU Report 241976 Determination of Absorbed Dose in a Patient Irradiated by Beams of X or Gamma Rays in Radiotherapy International Commission on Radiation Units and Measurements Bethesda, MD.

Karger C P, Jäkel O, Palmans H \& Kanai T 2010 Phys. Med. Biol. 55(21), R193.

Krämer M, Jäkel O, Haberer T, Kraft G, Schardt D \& Weber U 2000 Phys. Med. Biol. 45, 3299-3317.

Krämer M \& Scholz M 2000 Phys. Med. Biol. 45, 3319-3330.

Lühr A, Hansen D C, Jäkel O, Sobolevsky N \& Bassler N 2011a Phys. Med. Biol. 56, 2515-2533.

Lühr A, Hansen D C, Sobolevsky N, Palmans H, Rossomme S \& Bassler N 2011 b Acta Oncol. 50(6), 797805.

Lühr A, Toftegaard J, Kantemiris I, Hansen D C \& Bassler N 2012 Int. J. Rad. Biol. 88(1-2), 209-212.

Palmans H, Symons J E, Denis J M, de Kock E A, Jones D T L \& Vynckier S 2002 Phys. Med. Biol. 47(17), 3055.

Palmans H, Thomas R, Simon M, Duane S, Kacperek A, DuSautoy A \& Verhaegen F 2004 Phys. Med. Biol. 49(16), 3737.

Parodi K et al. 2007 Int. J. Radiat. Oncol., Biol., Phys. 68(3), 920 - 934.

Pshenichnov I, Botvina A, Mishustin I \& Greiner W 2010 Nucl. Instrum. Methods Phys. Res. B 268(6), 604-615.

Scholz M, Kellerer A M \& Kraft-Weyrather W 1997 Radiat. Environ. Biophys. 36, 59-66.

Sørensen B S, Overgaard J \& Bassler N 2011 Acta Oncologica 50(6), 757-762.

Toneev V \& Gudima K 1983 Nuclear Physics A 400, 173 - 189. 